\title{
Developing of Fuzzy Logic Controller for Air Condition System
}

\author{
Sameh Mohamed Sobhy
}

Wael Mohamed Khedr

\begin{abstract}
Fuzzy logic control was developed to control the compressor motor speed, fan speed, fin direction and operation mode to maintain the room temperature at or closed to the set point temperature and save energy and keep devices from damage. This paper describes the development of Fuzzy logic algorithm for Air Condition control system. This system consists of four sensors for feedback control: first for input electric volt which used to save devices from damage due to alternated voltages, second for temperature and third for humidity and fourth for dew point. Simulation of the Fuzzy logic algorithm for Air Condition controlling system is carried out based on MATLAB.
\end{abstract}

\section{Keywords}

Fuzzy Logic Controller (FLC), Fuzzy Inference Systems(FIS), and Air Conditioning System.

\section{INTRODUCTION}

The human brain can reasons with imprecise environments or decision based on uncertain or value judgment like the air is fine or the speed is fast or facts that are partially true. The fuzzy logic is a branch of machine intelligence that help computers to process and utilize vague data of humanistic systems [15]. Fuzzy inference systems (FIS) are information processing systems using fuzzy logic mechanism to represent the human reasoning process and to make decisions based on uncertain, imprecise environments. FIS consists of four parts fuzzfier, rules, inference engine, and defuzzfier as shown in the following [9]. To design FIS, needed the perioral experiences of human experts about field of research or knowledge base that observed and collected from operations of systems. Fuzzy logic manipulates such imprecise and vague data as fine or fast help engineering to controls and describes systems using commonsense rules that refer to indefinite quantities. So that it is possible to transition from one rule to another as the input is varied smoothly. These rules are linguistically natural representation of human's (or expert's) Knowledge base, that provides easy understanding knowledge representing scheme for explain information that has been learnt by a computer. For air condition system to manipulate temperature and the humidity close to an aimed value, and to save the electrical energy that taken by Air Condition compressor / Fan while utilizing all available resources in the most efficient manner [17]. Fuzzy logic system structure consists of database or prior knowledge that have to be crisp value to allow fuzzification using membership function, fuzzy control that manage fuzzification, rules evaluation, and defuzzification the output that also is crisp values as shown in figure (1).

1. Zagazig Uuniv., Faculty of Science, Math Dept.

2. Majmaah Uuniv., Faculty of Science, CSI Dept.

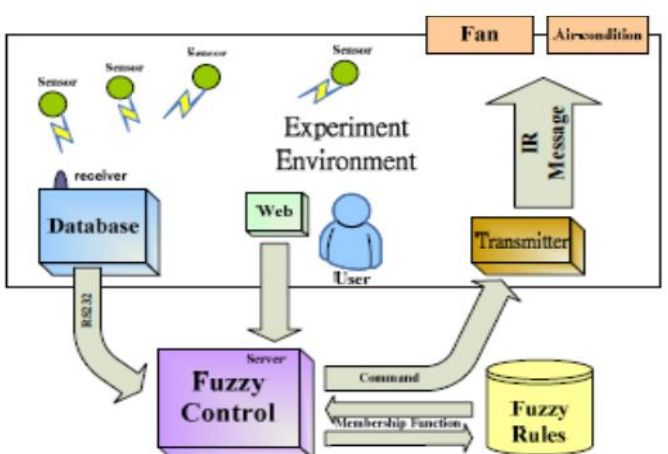

Figure 1.Fuzzy Logic system structure

To develop Air Condition system that control in humidity in own way without giving users any scope for changing the set point for the target humidity unlike the scope, it offer to change the set point for the target temperature through thermostat [24][25].That's leads to huge reducing energy using and providing necessary conditions for comfort living inside building. This system consists of four Sensors that captures temperature, Electric Volt, user temperature and humidity these reading are fuzzified figure 1 . These are used to decide the fuzzy qualifier, which is decoded into a crisp value that in turn controls different aspects of the Air Condition.

\section{AIR SYSTEM ORGANIZATION}

The dew point temperature determines what combinations of temperature and relative humidity $(\mathrm{RH})$ will be possible in the storage environment. At a constant dew point, when the temperature goes up, the RH goes down and when the temperature goes down, the RH goes up. Controlling the dew point is key to managing the risk of material decay. Used Dew Point Calculator that designed to calculate and visually present the relationship between temperature, relative humidity and dew point. These evaluation are used to evaluate the preservation quality of the environment [33]. Dew point temperature is used to measure humidity instead of relative humidity (RH). A standard Dew Point Human Reaction table are evaluated as in table (1).

\begin{tabular}{|c|c|}
\hline Dew Point & Reaction \\
\hline Above $20^{\circ} \mathrm{C}(68 \mathrm{~F})$ & Oppressive \\
\hline $18^{\circ} \mathrm{C}(64 \mathrm{~F})$ & Sticky \\
\hline $16^{\circ} \mathrm{C}(61 \mathrm{~F})$ & Humid \\
\hline $13^{\circ} \mathrm{C}(55 \mathrm{~F})$ & Comfortable \\
\hline $10^{\circ} \mathrm{C}(50 \mathrm{~F})$ & Refreshing \\
\hline Less than $10^{\circ} \mathrm{C}(50 \mathrm{~F})$ & Dry \\
\hline
\end{tabular}




\section{Table (1) : Human reaction to different levels of dew point}

Air Condition Fuzzy logic control System takes four variables into consideration showing in the following block:
(1) User temperature $\left(16^{\circ} \mathrm{C} \rightarrow 30^{\circ} \mathrm{C}\right.$ continuous control).
(2) Actual temperature.
(3) dew point temperature.
(4) Electricity Volt

User temperature subtracted from actual temperature before sending data for fuzzification step. Fuzzy arithmetic and criterion step is applied on these variables and final result is defuzzified step to get following crisp results as showing in the following figure (2):
(1) Compressor Speed
(2) Fan Speed.
(3) Mode of operation
(4) Fin Direction.

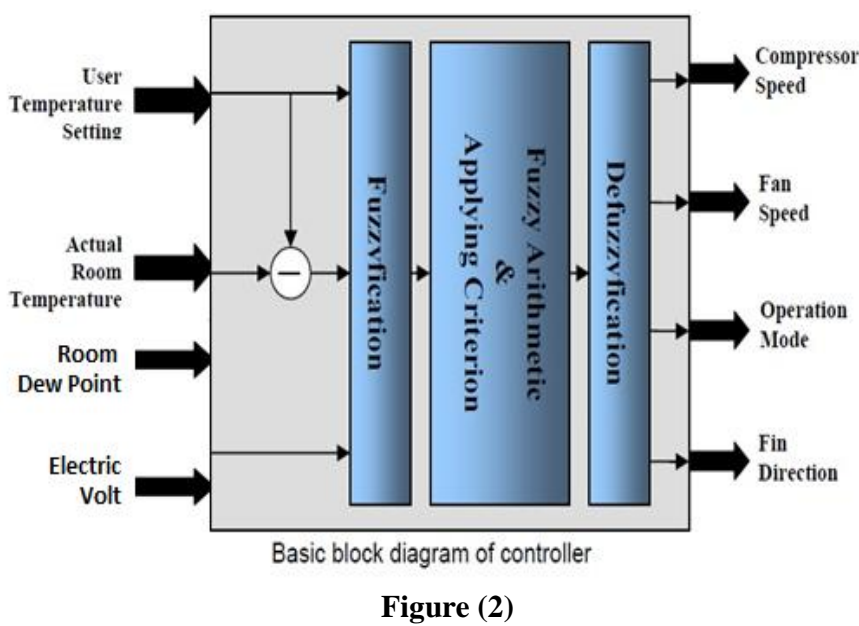

\section{FUZZY MEMBERSHIP FUNCTION}

The membership function editor in Fuzzy tool box is used to define the shapes of all membership functions associated with each membership variable $[9,10]$. In that system for each of the input and output variables the membership function is defined as follows

\subsection{Input Variables}

\subsubsection{User temperature (UT)}

users temperature that received by electronic, thermostat, are represented by three membership functions low, optimal, high as in table (2).

Table (2) : classification of user temperature (UT)

\begin{tabular}{|c|c|c|}
\hline Input field & Range & Fuzzy set \\
\hline \multirow{2}{*}{$\begin{array}{c}\text { User temperature } \\
(\text { UT) }\end{array}$} & $16-25$ & Low \\
\cline { 2 - 3 } & $22-28$ & Optimal \\
\cline { 2 - 3 } & $25-30$ & High \\
\hline
\end{tabular}

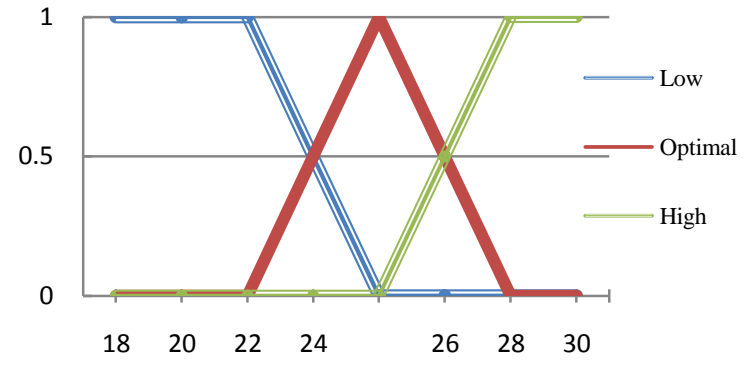

$\mu_{\text {low }}(\mathrm{x})=\left\{\begin{array}{cc}1 & , \quad x \leq 22 \\ \frac{25-x}{3} & , 22 \leq x \leq 25 \\ 0 & , \quad \text { other wise }\end{array}\right.$

$\mu_{\text {optimal }}(x)= \begin{cases}\frac{x-22}{3} & , 22 \leq x \leq 25 \\ \frac{28-x}{3} & , 25 \leq x \leq 28\end{cases}$

$\mu_{\text {high }}(\mathrm{x})=\left\{\begin{array}{cr}\frac{\mathrm{x}-25}{3} & , 25 \leq x \leq 28 \\ 1, & 28 \leq x \leq 30 \\ 0, & \text { other wise }\end{array}\right.$

\subsubsection{Temperature difference (Tdif)}

The different between room temperature actually and user temperature , are represented by four membership functions negative, zero, positive, large as in table (3).

Table (3) : Classification of Temperature Difference (Tdif)

\begin{tabular}{|c|c|c|}
\hline Input field & Range & Fuzzy set \\
\hline \multirow{4}{*}{ Temperature Difference (Tdif) } & $-1-0$ & Negative \\
\cline { 2 - 3 } & $-0.5-0.5$ & Zero \\
\cline { 2 - 3 } & $0-2$ & Positive \\
\cline { 2 - 3 } & $1-3$ & Large \\
\hline
\end{tabular}

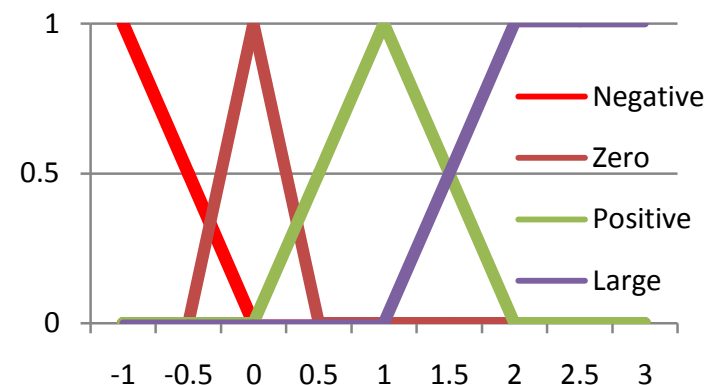

$$
\begin{aligned}
& \mu_{\text {negative }}(\mathrm{x})=\left\{\begin{array}{cc}
1 & ,-1 \leq x \leq-0.9 \\
-0.9 x & , \quad-0.9 \leq x \leq 0 \\
0 & , \quad \text { other wise }
\end{array}\right. \\
& \mu_{\text {zero }}(\mathrm{x})=\left\{\begin{array}{cc}
2(x+0.5) & , \quad-0.5 \leq x \leq 0 \\
2(0.5-x) & , 0 \leq x \leq 0.5
\end{array}\right. \\
& \mu \text { positive }(\mathrm{x})= \begin{cases}x & , 0 \leq x \leq 1 \\
2-x & , 1 \leq x \leq 2\end{cases} \\
& \mu_{\text {negative }}(x)=\left\{\begin{array}{cc}
1-x & , 1 \leq x \leq 2 \\
1 & , 2 \leq x \leq 3 \\
0 & \text { other wise }
\end{array}\right.
\end{aligned}
$$

\subsubsection{Dew Point (Td)}

Dew point describes information about dew point temperature inside the room. Using two membership functions optimal, humid as in table (4) . 
Table (4) : Classification of Dew Point (Td)

\begin{tabular}{|l|l|l|}
\hline Input field & Range & Fuzzy set \\
\hline \multirow{2}{*}{ Dew Point (Td) } & $10-14$ & Optimal \\
\cline { 2 - 3 } & $12-18$ & Humid \\
\hline
\end{tabular}

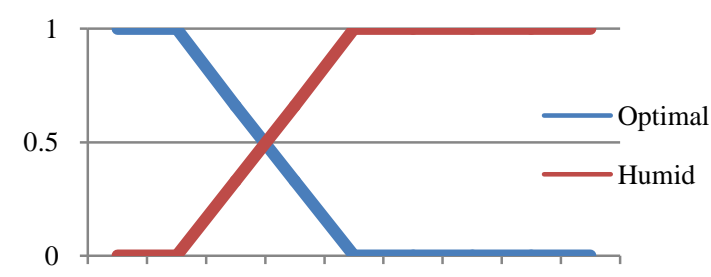

$\begin{array}{lllllllll}10 & 11 & 12 & 13 & 14 & 15 & 16 & 17 & 18\end{array}$

$\mu_{\text {optimal }}(\mathrm{x})=\left\{\begin{array}{cc}1 & , 10 \leq x \leq 11 \\ \frac{14-\mathrm{x}}{3} & , 11 \leq x \leq 14 \\ 0 & , \quad \text { other wise }\end{array}\right.$

$\mu_{\text {humid }} \quad(\mathrm{x})=\left\{\begin{array}{cc}\frac{x-12}{3} & , 12 \leq x \leq 15 \\ 1 & , 15 \leq x \leq 18 \\ 0 & , \text { other wise }\end{array}\right.$

\subsubsection{Electric Volt (EV)}

Electric volt describes the information about alternating volt that difference and then according to that difference by controlling in compressor and fan to stand by or work as rule listed. Using two membership functions low, regular as in table (5).

Table (5) : Classification of Electric Volt (EV)

\begin{tabular}{|l|l|l|}
\hline Input field & Range & Fuzzy set \\
\hline \multirow{2}{*}{ Electric Volt (EV) } & $130-180$ & Low \\
\cline { 2 - 3 } & $170-220$ & Regular \\
\hline
\end{tabular}

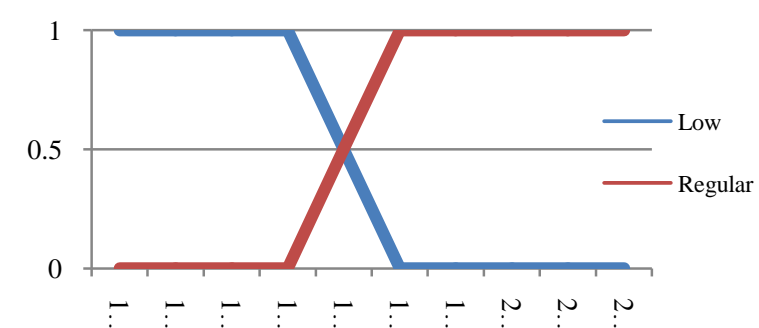

$\mu_{\text {low }}(\mathrm{x})=\left\{\begin{array}{cr}1 & , 130 \leq x \leq 160 \\ \frac{180-x}{20} & , 160 \leq x \leq 180 \\ 0 & , \quad \text { other wise }\end{array}\right.$

$\mu_{\text {regular }}(\mathrm{x})=\left\{\begin{array}{cc}\frac{\mathrm{x}-170}{20} & , 170 \leq x \leq 190 \\ 1 & , 190 \leq x \leq 220 \\ 0 & , \text { other wise }\end{array}\right.$

\subsection{Output membership function}

\subsubsection{Compressor Speed (SC)}

Compressor speed is characterized as low, medium and high different from 0 to $100 \%$.To evaluate the membership function (MF) by scaling the ranges from 0 to 100 based on speed. calculated MF and the range are given as in table (6).
Table (6) : Classification of Compressor Speed (SC)

\begin{tabular}{|l|l|l|}
\hline Input field & Range & Fuzzy set \\
\hline \multirow{3}{*}{ Compressor Speed (SC) } & $0-50$ & Low \\
\cline { 2 - 3 } & $40-80$ & Medium \\
\cline { 2 - 3 } & $70-100$ & Fast \\
\hline
\end{tabular}

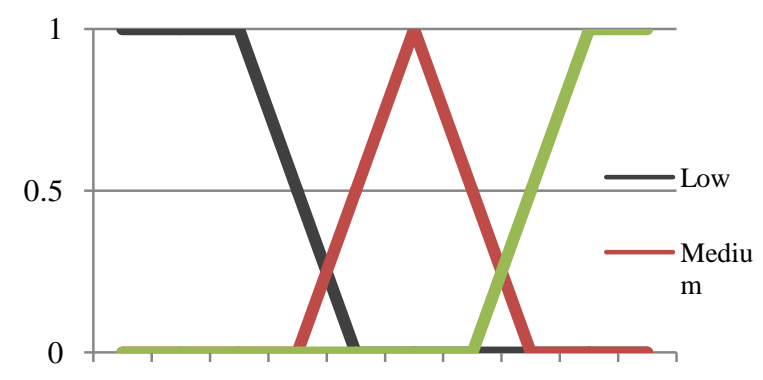

$\begin{array}{llllllllll}10 & 20 & 30 & 40 & 50 & 60 & 70 & 80 & 90 & 100\end{array}$

$\mu_{\text {low }}(\mathrm{x})=\left\{\begin{array}{cc}1 & , 0 \leq x \leq 30 \\ \frac{50-x}{20} & , 30 \leq x \leq 50 \\ 0 & , \text { other wise }\end{array}\right.$

$\mu$ medium $\quad(x)=\left\{\begin{array}{cc}\frac{x-40}{20} & , 40 \leq x \leq 60 \\ \frac{80-x}{20} & , 60 \leq x \leq 80 \\ 0 & , \text { other wise }\end{array}\right.$

$\mu_{\text {fast }}(\mathrm{x})=\left\{\begin{array}{cc}\frac{x-70}{20}, & 70 \leq x \leq 90 \\ 1 & , 90 \leq x \leq 100 \\ 0 & , \text { other wise }\end{array}\right.$

\subsubsection{Fan Speed $(F c)$}

Fan speed is characterized as low, medium and high varied from 0 to $100 \%$.To calculate membership function (MF) by scaling the ranges from 0 to 100 based on speed. Calculated $\mathrm{MF}$ and the ranges are given as in table (7).

Table (7) : Classification of Fan Speed (FC)

\begin{tabular}{|l|l|l|}
\hline Input field & Range & Fuzzy set \\
\hline \multirow{3}{*}{ Fan Speed (FC) } & $0-50$ & Low \\
\cline { 2 - 3 } & $40-80$ & Medium \\
\cline { 2 - 3 } & $70-100$ & Fast \\
\hline
\end{tabular}

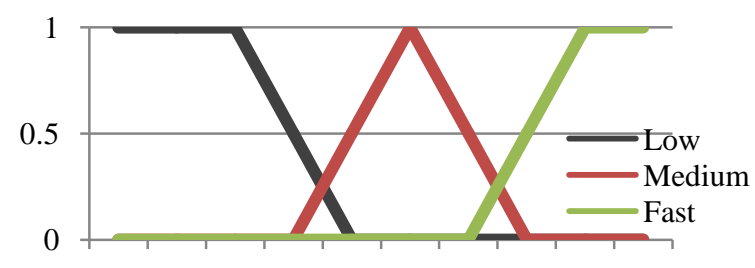

$\begin{array}{llllllllll}10 & 20 & 30 & 40 & 50 & 60 & 70 & 80 & 90 & 100\end{array}$

$\mu_{\text {low }}(\mathrm{x})=\left\{\begin{array}{cc}1 & , 0 \leq x \leq 30 \\ \frac{50-x}{20} & , 30 \leq x \leq 50 \\ 0 & , \text { other wise }\end{array}\right.$ 
$\mu$ medium $(\mathrm{x})=\left\{\begin{array}{cc}\frac{x-40}{20} & , 40 \leq x \leq 60 \\ \frac{80-x}{20} & , 60 \leq x \leq 80 \\ 0 & , \text { other wise }\end{array}\right.$

$\mu_{\text {fast }}(\mathrm{x})=\left\{\begin{array}{cc}\frac{x-70}{20}, & 70 \leq x \leq 90 \\ 1 & , 90 \leq x \leq 100 \\ 0 & , \text { other wise }\end{array}\right.$

\subsubsection{Mode of operation (Mo)}

Mode of operation decides whether air condition work like a dehumidifier only or normal.

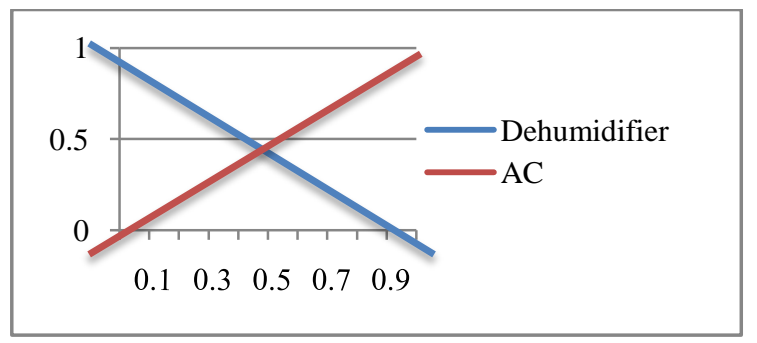

\subsubsection{Fin direction $(F n)$}

Fin directions directs air from air condition towards or away from occupants. Assuming top mounted air condition, $\theta=0^{\circ}$ can be considered as towards and $\theta=90^{\circ}$ as away from occupant[28,29].

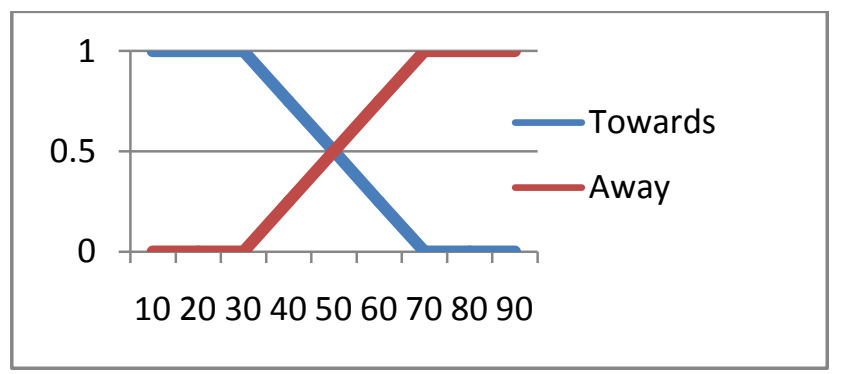

\section{FUZZY RULE BASE}

Fuzzy rules referred to as the IF-THEN rule base form and deductive form. And rules are based on natural language representation and models which are themselves based on fuzzy sets and fuzzy logic. It typically expressed inference like if the fact (premise, hypothesis, antecedent), then infer, or derive another fact called a conclusion. It design manual by a user, or automatic. The rules are defined by selecting the right sequence in the If-then sequence. It represent human empirical and heuristic knowledge in our language of communication that can be represented by fuzzy sets and logical connectivity of these sets . user temperature having three fuzzy ranges (low, optimal and high), temperature difference with four fuzzy ranges (negative, zero, positive and large), dew point with two fuzzy ranges (optimal and humid) and electric volt with two fuzzy ranges (low and regular) give a rule base matrix with size $3 * 4 * 2 * 2=48$ cells. Every cell has four outputs, each for compressor speed, fan speed, mode of operation and fin direction[11]. Fuzzy rules system are shown in table (8)

Table (8) : Fuzzy rules base

\begin{tabular}{|c|c|c|c|c|c|c|c|c|}
\hline \multirow{2}{*}{ 范 } & \multicolumn{4}{|c|}{ input variables } & \multicolumn{4}{c|}{ output variables } \\
\cline { 2 - 9 } & UT & Tdiff & Td & EV & CS & Fs & Mo & Fn \\
\hline 1 & Low & Negativ & optim & low & Low & Low & ac & Away \\
\hline
\end{tabular}

\begin{tabular}{|c|c|c|c|c|c|c|c|c|}
\hline 2 & Optim & Negativ & optim & low & Low & Low & ac & Away \\
\hline 3 & High & Negativ & optim & low & Low & Low & ac & Away \\
\hline 4 & Low & Zero & optim & low & Low & Low & $\mathrm{ac}$ & Away \\
\hline 5 & Optim & Zero & optim & low & Low & Low & $\mathrm{ac}$ & Away \\
\hline 6 & High & Zero & optim & low & Low & Low & $\mathrm{ac}$ & Away \\
\hline 7 & Low & Positiv & optim & low & Low & Low & $\mathrm{ac}$ & Away \\
\hline 8 & Optim & Positiv & optim & low & Low & low & ac & Away \\
\hline 9 & High & Positiv & optim & low & Low & Low & $\mathrm{ac}$ & Away \\
\hline 10 & Low & Large & optim & low & Low & Low & ac & Away \\
\hline 11 & Optim & Large & optim & low & Low & Low & ac & Away \\
\hline 12 & High & Large & optim & low & Low & Low & ac & Away \\
\hline 13 & Low & Negativ & optim & hig & Low & Low & ac & Away \\
\hline 14 & Optim & Negativ & optim & hig & Low & Low & $\mathrm{ac}$ & Away \\
\hline 15 & High & Negativ & optim & hig & Low & Low & ac & Away \\
\hline 16 & Low & Zero & optim & hig & Low & Fast & $\mathrm{ac}$ & Toward \\
\hline 17 & Optim & Zero & optim & hig & Low & medium & ac & Toward \\
\hline 18 & High & Zero & optim & hig & Low & Low & ac & Away \\
\hline 19 & Low & Positiv & optim & hig & Fast & Fast & ac & Toward \\
\hline 20 & Optim & Positiv & optim & hig & medium & medium & ac & Toward \\
\hline 21 & High & Positiv & optim & hig & medium & medium & ac & Toward \\
\hline 22 & Low & Large & optim & hig & Fast & Fast & ac & Toward \\
\hline 23 & Optim & Large & optim & hig & Fast & Fast & $\mathrm{ac}$ & Toward \\
\hline 24 & High & Large & optim & hig & Fast & Fast & ac & Toward \\
\hline 25 & Low & Negativ & humid & low & Low & Low & $\overline{a c}$ & Away \\
\hline 26 & Optim & Negativ & humid & low & Low & Low & ac & Away \\
\hline 27 & High & Negativ & humid & low & Low & Low & ac & Away \\
\hline 28 & Low & Zero & humid & low & Low & Low & ac & Away \\
\hline 29 & Optim & Zero & humid & low & Low & Low & $\mathrm{ac}$ & Away \\
\hline 30 & High & Zero & humid & low & Low & Low & ac & Away \\
\hline 31 & Low & positive & humid & low & Low & Low & ac & Away \\
\hline 32 & Optim & positive & humid & low & Low & Low & ac & Away \\
\hline 33 & High & positive & humid & low & Low & Low & $\mathrm{ac}$ & Away \\
\hline 34 & Low & Large & humid & low & Low & Low & $\mathrm{ac}$ & Away \\
\hline 35 & Optim & Large & humid & low & Low & Low & ac & Away \\
\hline 36 & High & large & humid & low & Low & Low & ac & Away \\
\hline 37 & Low & negativ & humid & hig & Fast & Fast & $\mathrm{de}$ & Toward \\
\hline 38 & Optim & negativ & humid & hig & Low & Low & $\mathrm{de}$ & Away \\
\hline 39 & High & negativ & humid & hig & Low & Low & de & Away \\
\hline 40 & Low & zero & humid & hig & Fast & Fast & $\overline{\mathrm{de}}$ & Toward \\
\hline 41 & Optim & zero & humid & hig & medium & Fast & $\mathrm{de}$ & Toward \\
\hline 42 & High & zero & humid & hig & medium & medium & $\mathrm{de}$ & Toward \\
\hline 43 & Low & positive & humid & hig & Fast & Fast & $\overline{a c}$ & Toward \\
\hline 44 & Optim & positive & humid & hig & Fast & Fast & ac & Toward \\
\hline 45 & High & positive & humid & hig & medium & Fast & $\mathrm{ac}$ & Toward \\
\hline 46 & Low & large & humid & hig & Fast & Fast & ac & Toward \\
\hline 47 & Optim & large & humid & hig & Fast & Fast & ac & Toward \\
\hline 48 & High & large & humid & hig & Fast & Fast & ac & Toward \\
\hline
\end{tabular}

\section{FUZZY LOGIC ALGORITHM}

Fuzzy logic algorithm is an algorithm that solves the problems expressed in the basic IF-THEN rule format. It consists of four steps as following:

Step 1 : linguistic Variables are the input variables of the system whose values are words or sentences from a natural language, instead of numerical values. A linguistic variable is generally decomposed into a set of linguistic terms.

Membership Functions Membership functions are used in the fuzzification and defuzzification steps of a FLS, to map the non-fuzzy input values to fuzzy linguistic terms and vice versa. A membership function is used to quantify a linguistic term.

Fuzzy Rules In a FLS, a rule base is constructed to control the output variable. A fuzzy rule is a simple IF-THEN rule with a condition and a conclusion.

Step 2 : Fuzzification means adding uncertainty by design to crisp sets or to sets that are already fuzzy and spreading the information provided by a crisp number or symbol to its vicinity so that the close neighborhood of the crisp number can be recognize by the computational tools. 
Step 3:Inference For each rule which represented in fuzzy level as set of restriction on the output based on certain conditions of the input. That restriction modeled by fuzzy set and relations and connected by linguistic connections like and, or, not and else, Obtaining the output which is a new fuzzy set which is the conclusion of rule since an implication operator is applied to the value of the antecedent obtained.

Step 4: defuzzification is the process of converting the result in fuzzy set form to a crisp result. It is important process for hardware application which process based on crisp data exchange. There is not theory to justify behavior of exchange other than commonsense reasoning such that the defuzzified output must represent a weight, voted, or must suitable solution. There are two main mechanism centroid method which based on finding a balance point of a property and maxima method which based in search for the highest pack whereas

\section{AIR CONDITION SYSTEM IMPLEMENTATION 6.1 Fuzzy Base Class}

Using Mamdani fuzzy system for the illustration which uses max aggregation and centroid method for deffuzification. The FIS Editor defines the Fuzzy Base Class, the various inputs, i.e. User temperature (ut), Temperature Difference (Tdiff), Dew Point (Td), and Electric Volt (EV) and the various output variables like Compressor Speed (Sc), Fan Speed (Sf), Mode of Operation (Mo) and Fin Direction (Fn) [10] as shown in Fig3 .

\subsection{Fuzzy Rule Base}

Fuzzy rules can be designed manually by a user, or automatically, i.e. the Rule Editor generates rules for all combinations of selected input variable and a user fills consequent fuzzy terms. Inputs and sub-sequentially provide the fuzzy outputs [10] as shown in fig 4.
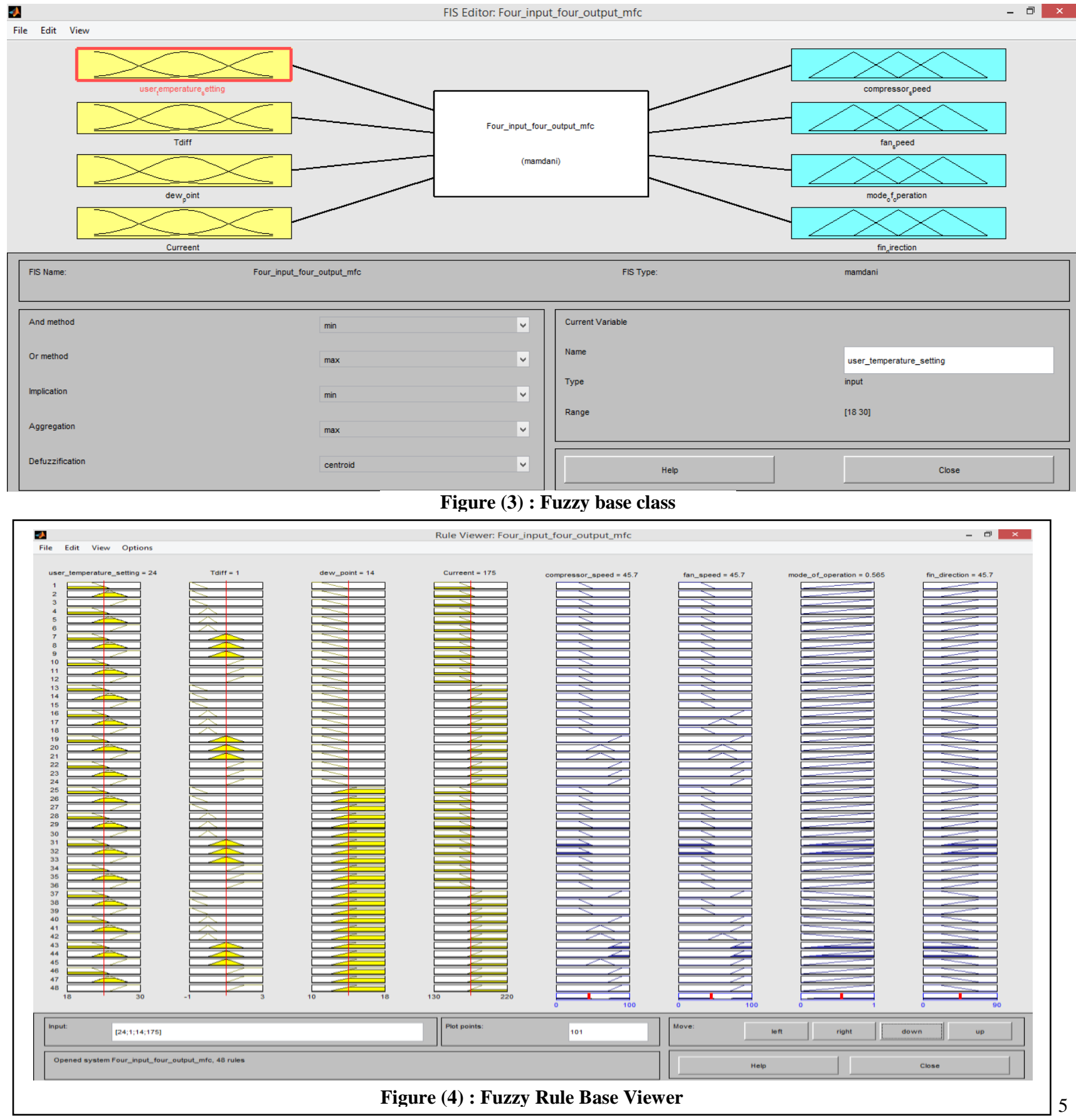


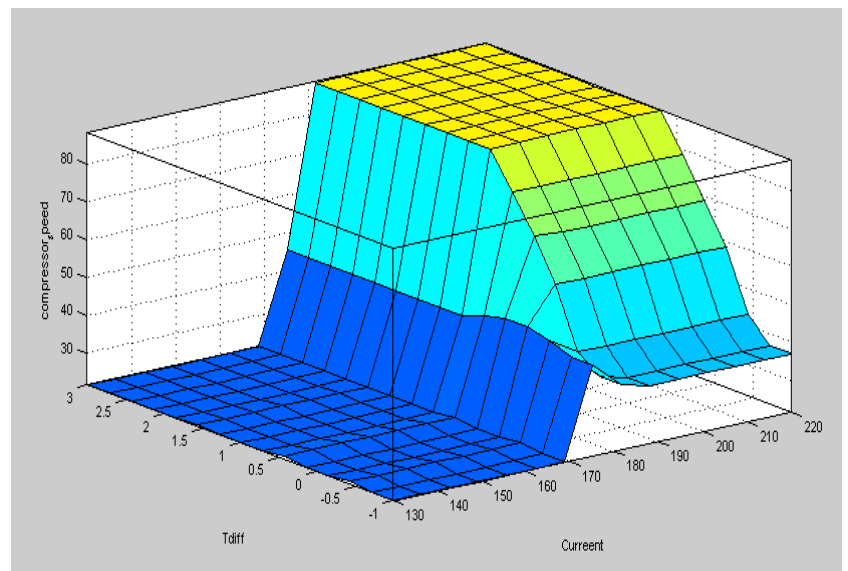

Figure (5) : Gradient Graph of Electric Volt vs. Temperature Diffrence vs compressor speed

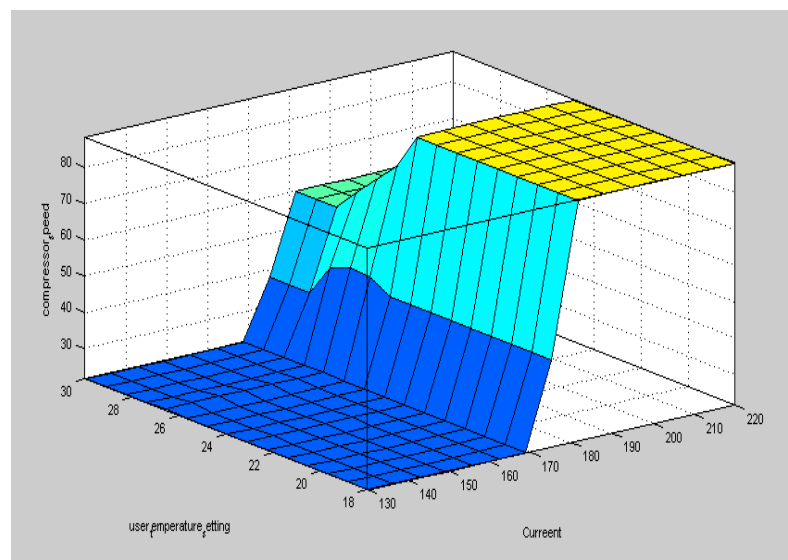

Figure (7) : Gradient Graph of Electric Volt vs. User Temperature vs. compressor speed.

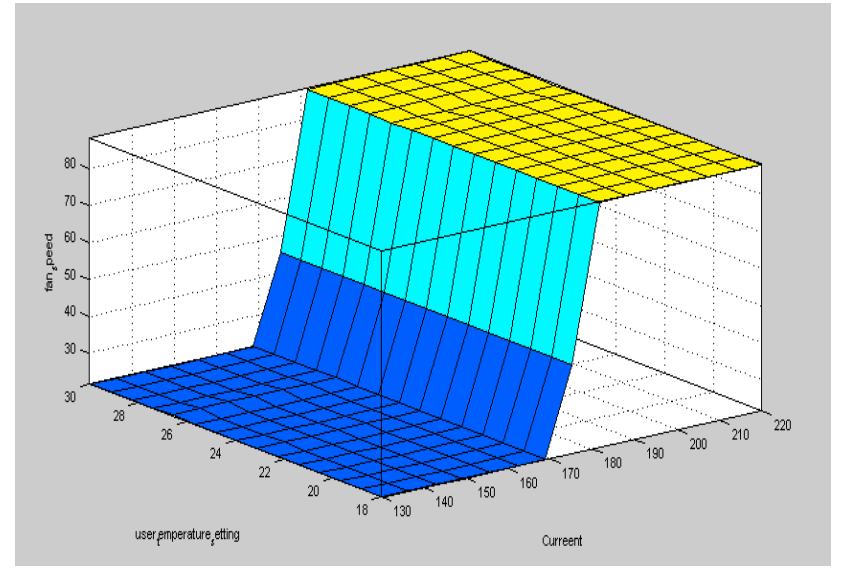

Figure (6) : Gradient Graph of Electric Volt vs. User Temperature vs. Fan speed

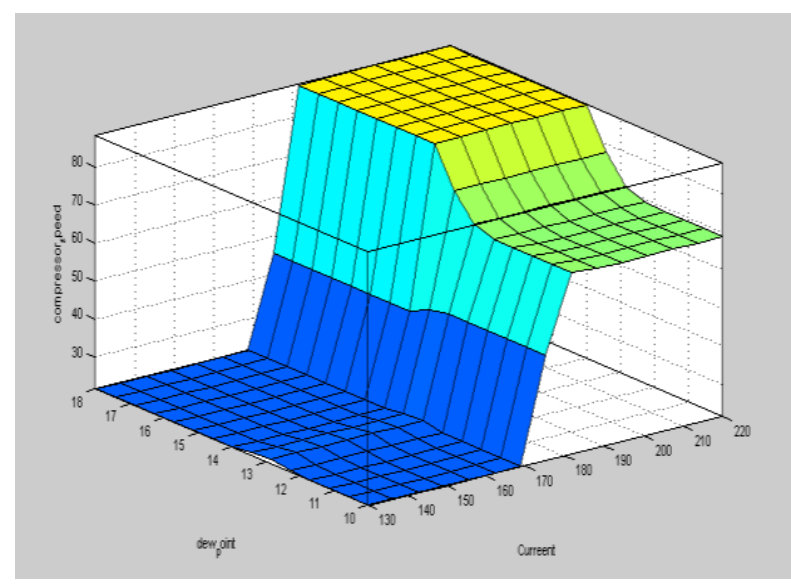

Figure (8) : Gradient Graph of Electric Volt vs.User Temperature vs. compressor speed. 


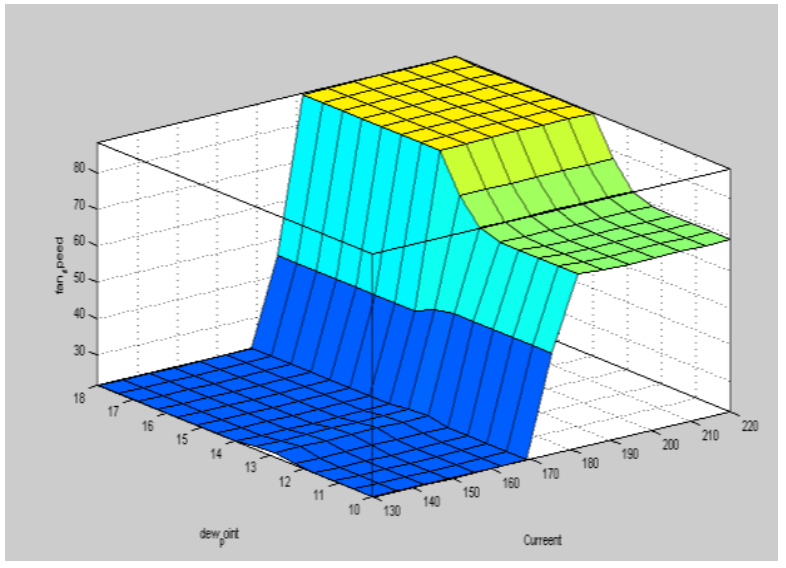

Figure (9): Gradient Graph of Electric Volt vc. Dew Point vc. Fan sneed

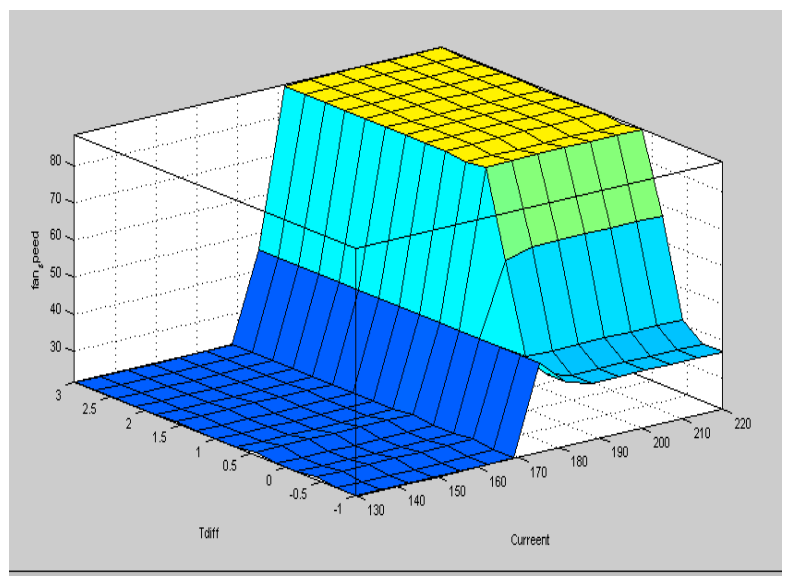

Figure (10) : Gradient Graph of Electric Volt vs. Temperature Difference vs. Fan

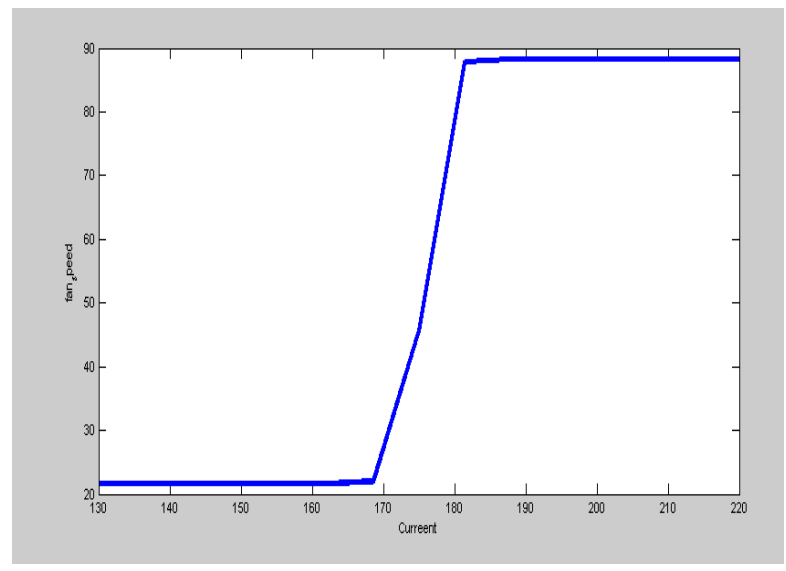

Figure (11) : Gradient Graph of Electric Volt vs. Fin speed

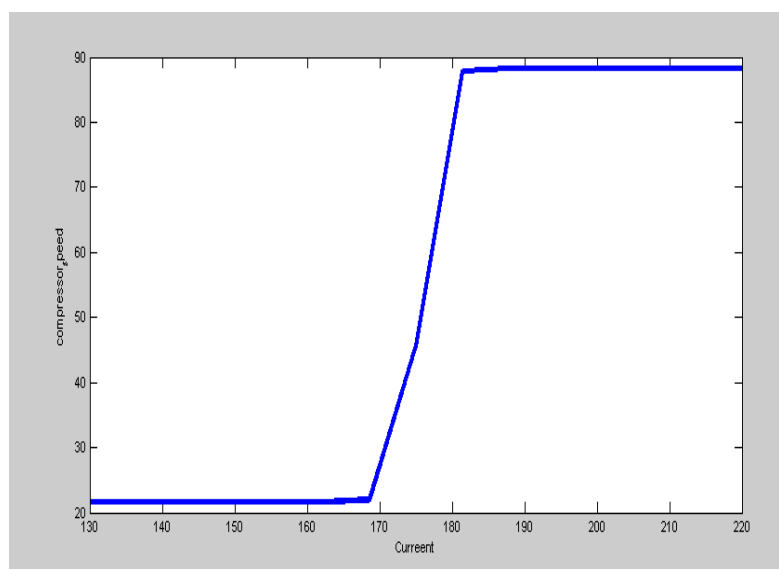

Figure (12) : Gradient Graph of Electric Volt vs. compressor speed

\section{CONCLUSIONS AND FUTURE WORK}

Previous Air Conditioning systems that are used to cool the rooms now can perform variety of functions. By adding intelligence to the system like fuzzy logic which is dealing with problems that are difficult and complex to study analytical that is now easy to solve in terms of linguistic variables. With most of the problems encountered in day to day life falling in this category, like washing machines, vacuum cleaners, etc., fuzzy logic is sure to make a great impact in human life. The neural net acts like computer because it maps input to output the neurons and synapses may be silicon component or equations in software that simulate their behavior. Supervised networks tune the rules of fuzzy system as if they were synapses. We will use neural network that can help fuzzy systems learn rules which can accepts pairs of input and output data and cluster them in a small number of classes.

\section{REFERENCES}

[1] Karray FO, de Silva C (2004) Soft computing and intelligent system design. Addison Wesley Longman, Boston.

[2] Zadeh LA (1965) Fuzzy sets. Info Control 8(3):338-353.

[3] Guanrong C, Trung P (2000) Introduction to fuzzy sets, fuzzy logic, and fuzzy control systems CRC, Boca Raton, FL.

[4] Timothy RJ (1995) Fuzzy logic with engineering applications. McGraw-Hill, Boston.

[5] J. Mendel. Fuzzy logic systems for engineering: a tutorial. Proceedings of the IEEE, 83(3):345\{377, Mar 1995.

[6] Buckley , J ; Tucker, D. (1995) - Second generation fuzzy expert system- Fuzzy Sets and Systems.

[7] Forgy. C.L. (2002). On the efficient implementation of production systems, $\mathrm{PhD}$ thesis- Department of computer science CMU.

[8] Graham. I . (2000) - Fuzzy logic in commercial expert systems results and prospects Fuzzy Sets and Systems).

[9] Jan Jantzen (1998),’Tutorial on fuzzy logic”. 
[10] Lotfi A (2001), Zadeh. "Fuzzy logic Toolbox",

[11] Lotfi A.Zadeh (1999),"Fuzzy systems", Handbook, Second Edition.

[12] Mamdani E. H. (2000) - Application of fuzzy logic to approximate reasoning using guistic synthesis. IEEE Transaction on Computers .

[13] Rebecca Shalfield (2001),"Fuzzy logic toolkit “, http://www.Ipa.co.uk/.

[14] Zadeh L. A (1998) -The role of fuzzy logic in the management of uncertainty in expert system- Fuzzy Sets and Systems - North Holland.

[15] Allahverdi N, "Expert Systems, An Application of Artificial Intelligent", Atlas Press, Istanbul, 2002.

[16] Tsoukalas L. H, Uhrig R. E, "Fuzzy and Neural Approaches in Engineering", John Wiley \& Sons, Inc. New York, USA, 1997.

[17] Wakami N, Araki S, Nomura H, "Recent Applications of Fuzzy Logic to Home Appliances", Proceedings of the IECON '93. International Conference on, 15-19 Nov. 1993, vol.1, pp. 155-160, 1993.

[18] Caponetto, R, Fortuna, L, Nunnari, G, Occhipinti, L, “A fuzzy approach to greenhouse climate control" American Control Conference, Proceedings of the 1998, vol.3, pp.1866- 1870, 1998.

[19] Pan Lanfang, Wang Wanliang, Wu Qidi, "Application of adaptive fuzzy logic system to model for greenhouse climate" Intelligent Control and Automation, Proceedings of the 3rd World Congress on vol. 3, pp.1687-1691, 2000.

[20] Orchard, B., "Fuzzy Sets", http://www.iit.nrc.ca/IR public/fuzzy/fuzzyJDocs/ FuzzySet.html, Accessed April 2006.

[21] Agarwal, S., Joshi, A., Finin, T. and Yesha, Y., "A Pervasive Computing System for the Operating Room of the Future", http://ebiquity.umbc.edu/get/a/ publication/328.pdf, Accessed April 2006.

[22] Casas, F., A. Orozcob, W.A. Smitha, J.A. De AbreuGarc1'ab, J. Durkin, "A fuzzy system cardio pulmonary bypass rotary blood pump controller", Expert Systems with Applications 26, pp. 357-361, 2004.
[23] Fuzzy control programming. Technical report, International Electrotechnical Commission, 1997.

[24] US Patent - 5,921,099; Air conditioner temperature control apparatus; Inventor: Seon Woo Lee; Assignee: Samsung Electronics Co., Ltd. Issue date: Jul 13, 1999.

[25] US Patent - 5,148,977; control system for air conditioner; Inventors: Yozo Hibino, Susumu Nakayama, Hiromu Yasuda, Kensaku Oguni, Kenji Tokusa; Assignee: Hitachi, Ltd. Issue date: Sep 22, 1992.

[26] Technical case studies and articles on fuzzy logic and fuzzy logic based control systems www.sciencedirect.com, http://en.wikipedia.org and http://www.aptronix.com/

[27] X.M. Song, "Research on LQR-fuzzy control algorithm of inverted pendulum system", Xi'an University of Electronic Science and Technology Master's thesis, January 2006

[28] Amiya Patanaik, " Fuzzy Logic Control of Air Conditioners", Indian Institute of Technology, Kharagpur, - 721302, India.

[29] Sanjit Kumar Dash, Gouravmoy Mohanty, Abhishek Mohanty," Intelligent Air Conditioning System using Fuzzy Logic", International Journal of Scientific \& Engineering Research Volume 3, Issue 12, December2012

[30] K. Lavanya1, M.A. Saleem Durai2, N.Ch. Sriman Narayana Iyengar3," Fuzzy Rule Based Inference System for Detection and Diagnosis of Lung Cancer", International Journal of Latest Trends in Computing ( $E$ ISSN: 2045-5364) 165 Volume 2, Issue 1, March 2011

[31] M. GLORIA SANCHEZ-TORRUBIA, CARMEN TORRES-BLANC, SANJAY KRISHNANKUTTY," Mamdani's Fuzzy Inference eMathTeacher: a Tutorial for Active Learning",

[32] Kuntze, H.-B, Bernard, Th, "A new fuzzy-based supervisory control concept for the demand-responsive optimization of HVAC control systems" Decision and Control, Proceedings of the 37th IEEE Conference on, vol. 4, pp. 4258-4263, 1998.

[33] http://www.dpcalc.org/index.php. available at:dew point calculator. 